\title{
Role of Fluid-Mediated Interactions in Guiding Nanoparticle Assembly
}

Utkur. Mirsaidov ${ }^{1,2,3 *}$ Guanhua Lin ${ }^{1,2,3}$, Duane Loh ${ }^{1,2}$, Jingyu Lu ${ }^{1,2,3}$, Zainul Aabdin ${ }^{1,2,3}$

${ }^{1 .}$ Centre for Advanced 2D Materials and Graphene Research Centre, Department of Physics, National University of Singapore, 6 Science Drive 2, Singapore 117546.

2. Center for BioImaging Sciences, Department of Biological Sciences, National University of Singapore, Singapore.

3. Nanocore, 4 Engineering Drive 3, National University of Singapore, Singapore 117576,

The assembly process of nanostructures from nanoparticles in solution is fundamental for "bottom-up" fabrication of functional materials and devices. The collective behaviour of these nanoparticle assemblies can give rise to new optoelectronic (1), electrochemical (2) and magnetic properties (3) different from the bulk or individual nanoparticles.

Using dynamic in situ TEM imaging (3-6) in liquids, we show the nanoparticle-nanoparticle interaction in thin fluid layer. We extend on our recent study (7) of nanoparticle bonding to show the effect of attractive hydration and depletion forces (in the case of small molecules in solution) which arise due to a single to double layers of water molecules separating these interacting nanoparticles. Using a statistical method, we probe the strength of both short-range and long-range inter-particle interactions and empirically derive the nanoparticle-nanoparticle interaction potential.

Furthermore, we will show that interaction of nanoparticles with liquid-liquid interfaces in the case of phase separated nandroplets in solution can be used for self-assembly. Our observation of the assembly dynamics of nanoparticles at fluid-fluid interface around the dispersed fluid-like ethylenediaminetetraacetic acid (EDTA) nanodroplets in solution reveal that particles bind to interface irreversible until the interface is fully populated. Our findings reveal that nanoscale capillary force is the main driver of nanoparticle arrangement and organization at fluid-fluid interfaces. We find that nanoparticles assemble into rings around dispersed nanodroplets in solution through: (i) direct attachment to the fluid-fluid interface, (ii) insertion of new nanoparticles between interface-bound nanoparticles, and (iii) coalescence of assemblies (Fig. 1).

Our findings show that the effect of hydration and capillary forces on assembly is one of many interactions that need to be understood for assembly of hierarchical nanostructures from nanoparticles serving as individual building blocks.

\section{References:}

[1] K. L. Kelly, E. Coronado, L. L. Zhao, G. C. Schatz, The Journal of Physical Chemistry B 107 (2002), p. 668.

[2] Y. Yamada et al., Nature Chemistry 3 (2011), p. 372.

[3] G. Singh et al., Self-assembly of magnetite nanocubes into helical superstructures. Science $\mathbf{3 4 5}$ (2014), p. 1149.

[4] M. J. Williamson, R. M. Tromp, P. M. Vereecken, R. Hull, F. M. Ross, Nature Materials 2 (2003), p. 532.

[5] H. Zheng, R. Smith, Y. Jun, C. Kisielowski, U. Dahmen, A. P. Alavisatos, Science 324 (2009), p. 1309.

[6] U. Mirsaidov, H. Zheng, D. Bhattacharya, Y. Casana, P. Matsudaira, Proc. Natl. Acad. Sci. U.S.A. 109 (2012), p. 7187.

[7] Z. Aabdin, J. Lu, X. Zhu, U. Anand, D. Loh, H. Su, U. Mirsaidov, Nano Letters 14 (2014), p. 6639. 
[8] This work was supported by the Singapore National Research Foundation's Competitive research program funding (NRF-CRP9-2011-04).
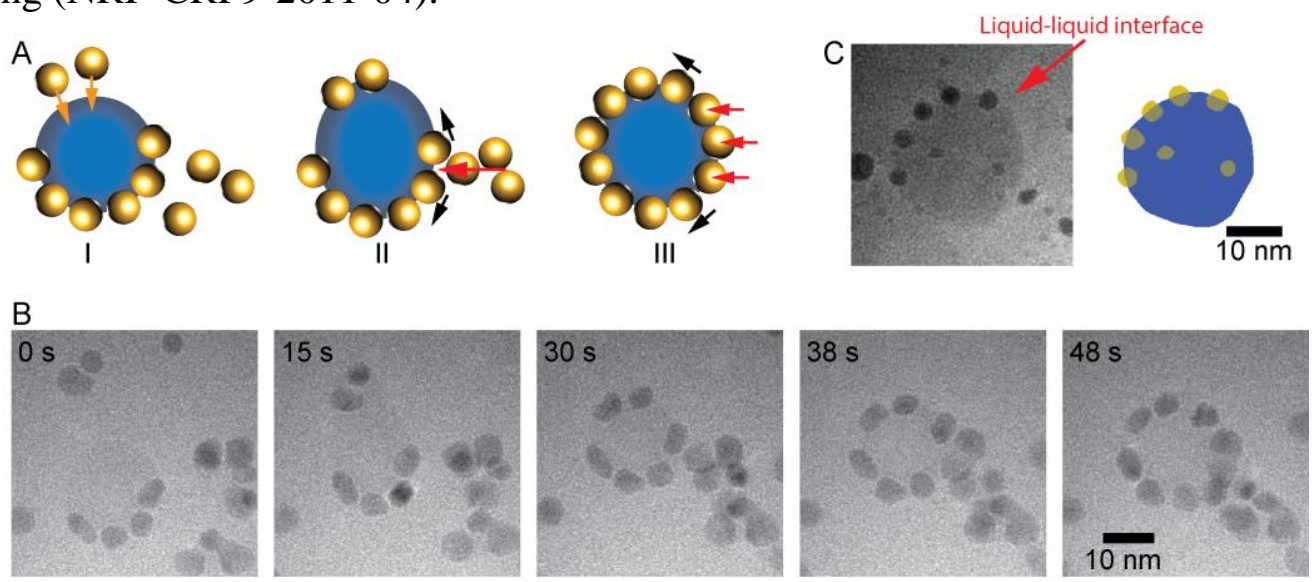

Figure 1. Graphene nanochannel platform. (A) Schematic illustration of binding dynamics of $\mathrm{Pt}$ nanoparticles to a nanodroplet by direct attachment (yellow arrows) to the circumference and by insertion (red arrow) between two closely spaced nanoparticles that are bound to nanodroplet circumference (blue arrow) in solution. (B) TEM image time series of binding dynamics of Pt nanoparticles to a nanodroplet circumference by direct attachment in solution. (C) TEM image and corresponding segmentation of Pt nanoparticles (yellow) assembled around the EDTA nanodroplet (blue) in water. 